\title{
LA AVANZADA MATERIAL DEL VIEJO IDEALISMO
}

\author{
MARÍA J. BINETTI \\ CONICET - Argentina
}

\begin{abstract}
RESUMEN: El presente artículo se proponer trazar la línea de continuidad especulativa que une al idealismo absoluto con lo que algunos autores denominan el último programa materialista del viejo idealismo alemán. El giro ontológico o especulativo del pensamiento contemporáneo se ha proclamado heredero de la vieja tradición idealista, especialmente en consideración de dos ideas centrales, a saber: la negatividad reflexiva del absoluto y su inmanencia medial en el fundamento de todas las cosas. Sobre estas dos ideas se articula el nuevo materialismo dialéctico, cuyos lineamientos generales intentaremos seguir, en particular a través de Slavoj Žižek y Catherine Malabou.
\end{abstract}

PALABRAS CLAVE: Giro especulativo; ontología; dialéctica; negatividad; mediación; ecología.

\section{The material Advance of the old Idealism}

ABSTRACT: The current article aims at drawing the line of speculative continuity that connects the absolute idealism with what some authors have called the last materialist program of the old German idealism. The ontological or speculative turn of contemporary philosophy has been proclaimed itself as heir to the old idealist tradition, especially in consideration of two main ideas, namely: the reflexive negativity of the absolute, and its mediate immanence in the ground of everything. Around these two ideas articulates the new dialectical materialism, which general features I will try to follow, particularly through Slavoj Žižek, and Catherine Malabou.

KEY WORDS: Speculative turn; ontology; dialectics; negativity; mediation; ecology.

\section{INTRODUCCIÓN HISTÓRICA AL CONCEPTO DE «MATERIA»}

El concepto de «materia» remonta su etimología a la prehistoria de un dinamismo matricial autopoiético y refiere su semántica al vitalismo de la conciencia humana primitiva. Desde entonces, ha recorrido toda la historia del pensamiento universal con las ambigüedades, equívocos y avatares que cualquier categoría esencial padece a lo largo de los tiempos.

La materia prehistórica es simbolizada en la madera de aquel ancestral árbol de la vida, emplazado en los jardines más remotos de la conciencia. Tanto la hyle griega - bosque, madera, material de construcción- como la materia latina - la sustancia de la mater, la matriz, el tronco productor de retoños- derivan su sentido de un núcleo generador de vida, potencia activa, creadora y expansiva, que permanece en continua transformación. Eugenio Trías recuerda al respecto que el significado original de la materia es el de una potencia activa, positiva e insondable, cuya irrupción produce y alimenta el $\operatorname{cosmos}^{1}$. En el amanecer de la filosofía occidental, el arché de los presocráticos da cuenta de esa materialidad autopoiética,

1 TRíAs, E., La edad del espíritu, DeBolsillo, Barcelona 2006, pp. 62 ss. 
concebida como principio, elemento y fin de la vida universal, medida de orden e inteligibilidad. El origen y elemento material de estos primeros filósofos es vivo, inteligente y divino, inengendrado, incorruptible y en continua transformación cíclica y circular. Todo nace de él y a él todo retorna en su disolución.

Entre el hilozoísmo presocrático y el espiritualismo platónico-aristotélico se ubica el dualismo órfico-pitagórico, anclado en el ideal ascético-religioso de un espíritu puro, con lo cual el sentido activo y generador de la materia fue trastocado en impotencia, degeneración y caída. A partir de entonces, la historia de la filosofía reemplazó el hilozoísmo de la conciencia primitiva por un dualismo espiritualista de raigambre ascético-religiosa, en cuyo contexto la materia pasó a significar pasividad, receptividad y privación ininteligible, cuando no corrupción y copia degradada. Ya se trate de la caverna platónica, el paradigma hilemórfico, el dualismo cartesiano o los construccionismos lingüísticos y culturales contemporáneos, todos ellos comulgan en la pasividad e inoperancia per se de la materia, entregada a una acción formadora de índole inmaterial, ya sea espiritual o histórico-cultural.

Sin embargo, junto con esta suerte de oscurantismo material dominante, han subsistido siempre resabios de aquella otra concepción primitiva de la materia como sustrato vivo y creador, presentes tanto en las doctrinas materialistas como en los vitalismos panteístas de todos los tiempos. Durante la modernidad, esta concepción autoactiva de la materia fue promovida por la nueva ciencia, en particular por la física newtoniana, que vino a confirmar la fuerza de resistencia y conservación ínsita en la materia. Misticismo panteísta, vitalismo universal y ciencia moderna confluyeron en la formación de un nuevo paradigma material, representado por la conciencia romántico-idealista y su concomitante resignificación del mundo natural como expresión divina.

Al idealismo romántico de un universo material divinizado, se remonta hoy el giro especulativo y materialista de autores como Maurizio Ferraris, Slavoj Žižek, Catherine Malabou, Adrian Johnston, Markus Gabriel, Jane Bennett, Iain Hamilton Grant, Rosi Braidotti o Elizabeth Grosz, entre otros. Muchos de ellos se han proclamado herederos de la vieja tradición idealista, en especial atención de dos ideas centrales, a saber: la negatividad reflexiva del absoluto y su inmanencia medial en el fundamento de todas las cosas. El común denominador de ambas corrientes consistiría entonces en el acceso ontológico a un absoluto material, negativo y medial.

Los párrafos que siguen se proponen trazar la línea de continuidad especulativa que une al idealismo absoluto con lo que Adrian Jonhston ha denominado «el último programa sistemático del idealismo alemán» ${ }^{2}$. Procederemos a partir de los primeros románticos hacia el idealismo de Friedrich W. J. Schelling y Georg W. F. Hegel, y desde estos últimos hasta el materialismo dialéctico de Slavoj Žižek y Catherine Malabou, tomados como ejemplos de una de las más promisorias tendencias contemporáneas.

2 Johnston, A., Adventures in Transcendental Materialism. Dialogues with Contemporary Thinkers, Edinburgh University Press 2014, pp. 23, 13. 


\section{El HILOZOÍSMO ROMÁNTICO}

Si se piensa en aquellos antiguos cosmólogos jónicos enamorados de la naturaleza, para quienes la materia era principio de vida y el universo entero estaba lleno de dioses, o si se evoca el sentimiento panteísta de esos místicos medievales cuya divinidad animaba todas las cosas, entonces podría verse en los jóvenes románticos de fines del siglo XVIII cierta reedición de ese eterno naturalismo, que Friedrich Schlegel ha denominado un "panteísmo de la naturaleza» ${ }^{3}$. El mundo encantado de los románticos con sus bosques y animales fabulosos, las montañas y los palacios de sus leyendas, las hadas, los elfos y esos espíritus elementales escondidos debajo de las piedras, los misterios y terrores de sus profundidades subterráneas, ellos son algunos de los tantos símbolos a través de los cuales el primer romanticismo alemán poetizó la intuición ontológica de una misma vida universal fluyendo por las venas de todas las cosas.

En el origen especulativo del romanticismo confluyen el misticismo de Jacob Boehme, el monismo spinoziano -interpretado por Johann G. Herder como un panteísmo vitalista - y el actualismo de la autoconciencia fichteana, a lo cual podría añadirse la nueva física newtoniana. Respecto de nuestro particular interés en torno a la cuestión material, la confluencia de tales ideas dio por resultado la concepción romántica de la materia como fuerza activa y creadora. La resignificación no dualista de la materia misma, concebida ahora como sustrato vivo y dinámico, se tradujo en la concepción del mundo natural como un organismo vivo, teleológicamente ordenado ${ }^{4}$. El universo romántico vive y respira como un organismo infinito, uniendo y separando a la vez cada uno de sus miembros y elementos.

La puesta en valor romántico-idealista del mundo material y natural transparenta el dinamismo especulativo de su absoluto inmanente, reflexivo y medial. Mientras que el monismo metafísico — de raíz mística y spinoziana — tramitaba la superación del sustancialismo dualista, el dinamismo reflexivo y medial del absoluto herencia del sujeto fichteano- suponía una identidad diferencial y negativa, capaz de unir y separar simultáneamente — es decir, de mediar — todos los contrarios. En este nuevo concepto de identidad reside precisamente la revolución especulativa del romanticismo, resumida por Frederick C. Beiser en el intento de «lograr la identidad-en-la-diferencia, la unidad-en-la-oposición» ${ }^{5}$. Lo absoluto no es ninguno de los términos abstractos, sino el medio, el "entre», el centro y círculo de un proceso en continua transformación.

La nueva era especulativa iniciada por los primeros románticos alemanes, respecto de la cual la identidad vale como diferencia absoluta y viceversa, supuso la emergencia de las dos grandes categorías que articularán en lo sucesivo el concepto de materia, a saber: negatividad y mediación. El concepto de negatividad, entendido por la metafísica clásica como opuesto dualista y excluyente de lo positivo, adquirirá ahora una consistencia afirmativa y dinámica por el hecho

3 Schlegel, F., Dialogue on Poetry and Literary Aphorism, trad. e intr. Ernst Behler y Roman Struc, The Pennsylvania State University Press, Pennsylvania 1968, p. 27.

4 Cf. BeIser, F. C., The Romantic Imperative. The Concept of Early Romanticism, Harvard University Press, Cambridge \& London 2003, pp. 182-86.

$5 \quad$ Ibid., p. 33. 
de pertenecer a la identidad reflexiva y dialéctica del absoluto. En cuanto a la mediación, su concepto explica justamente la valencia afirmativa de la negatividad, en la medida en que lo real no comporta la simple inmediatez sino el desdoblamiento y la actualidad recíproca de la diferencia en el seno de la creación absoluta. Junto con la resignificación medial de lo negativo, el romanticismo revaloriza todos aquellos conceptos y símbolos tradicionalmente implicados con él, por ejemplo: la oscuridad, el caos, la noche, la muerte y particularmente en lo que nos atañe, la materia y el mundo natural, recuperados ahora en el dinamismo superior de un absoluto medial.

El proyecto romántico-idealista de un hilozoísmo universal debe ser comprendido en el seno de este absoluto reflexionado — negado y mediado- en y por la materialidad de su esencia. El mundo material inaugura el tiempo y el espacio del absoluto, manifiesta su vida divina, corporiza su logos y lenguaje. La inmanencia perfecta del monismo romántico significa que no hay trascendencia alguna más allá de esta existencia material y natural, donde el absoluto se realiza a sí mismo como tiempo, espacio y finitud. De aquí que el alma romántica se reconozca a sí misma en el mundo natural como en un espejo, que le devuelve su propia imagen poetizada. Lo más íntimo de la subjetividad romántica existe y se mueve en ese poema natural que la revela.

Los románticos alemanes son discípulos de los naturalistas y los místicos ${ }^{6}$. En ellos vibra una simpatía cósmica que los hermana con el universo entero. Naturaleza y subjetividad constituyen lo cóncavo y convexo de la unidad divina y de allí su conexión esencial, su idéntico resonar armónico, del cual deriva una suerte de ethos material, naturalista o medioambiental que parece adelantarse a la conciencia ecológica contemporánea. Novalis explica al respecto que el sentimiento moral corresponde a la aprehensión de la totalidad creadora y de allí la convergencia entre naturaleza, ética y creación: «el sistema de la moral debe convertirse en el sistema de la naturaleza [...] La naturaleza llegará a ser moral» ${ }^{7}$. Con esta afirmación, el romanticismo da por supuesta la reciprocidad de una acción libre, devenida materia y espíritu, naturaleza y cultura a la vez.

En el marco de este absoluto negativo y medial, se presenta el concepto de materia como base de la filosofía de la naturaleza romántica. Por el hecho de ser concebida en la mediación esencial del absoluto, se trata eo ipso de una categoría ontológico-especulativa, cuyo tratamiento más sistemático se les debe a Schelling y a Hegel, a quienes nos referiremos a continuación. Ambos son receptivos de la tradición científica de su época, previamente elaborada por el análisis trascendental kantiano de la materia como fuerza de atracción y repulsión, en el que ellos vieron la superación de la mecánica newtoniana. A la física dinámica de Kant, tanto Schelling como Hegel le opondrán una física especulativa, especificada por la inmanencia vitalista del romanticismo.

Schelling comprendió rápidamente el alcance que la superación romántica del sustancialismo dualista tenía respecto de la restitución a «la esencia y dignidad de

6 Cf. Béguin, A., El alma romántica y el sueño, trad. Mario Monteforte Tolero, FCE, Madrid 1978, p. 99.

7 Novalis, Los fragmentos. Los discípulos en Sais, introd. y trad. Mauricio Maeterlinck, El Ateneo, Buenos Aires 1948, p. 119. 
la materia ${ }^{8}$, esencia y dignidad que milenios de falogocentrismo habían denostado bajo la representación abstracta de un mero receptáculo vacío. El nuevo absoluto medial determinó la identidad espíritu=materia y con esto, explica Schelling, "se pone término a una cantidad de investigaciones desorientadoras sobre la relación entre ambos ${ }^{9}$. Ni materia ni espíritu serán a partir de ahora sustancias simples, claras y distintas, jerárquicamente ordenadas como pasividad y actualidad respectivas, sino un mismo sujeto en su devenir reflexivo y medial, de donde preguntar cuál de ellas tiene la primacía resulta tan absurdo como preguntar por la prioridad del huevo o la gallina. En palabras de Schelling, «la materia no es más que el espíritu intuido en el equilibrio de sus actividades», una especie de «espíritu derramado (geronnenen) ${ }^{10}$ a la percepción sensible.

Con el concepto schellingiano de materia se establece la categoría central de su idealismo, a saber, la noción de "potencia» como término medio entre el ser y el no-ser, constitutivo de la negatividad reflexiva del absoluto romántico-idealista. Schelling explica al respecto que «hay algo medio entre lo que es y la nada: lo que no es ni debe ser, pero intenta ser ${ }^{11}$. La potencia, el poder, lo posible definen el origen y fundamento de lo real, y a esto se refiere la materia schellingiana en tanto que «negación originaria» $\mathrm{o}$ "potencia negadora» ${ }^{12}$, reiteradamente descrita como «la madre y la nodriza de todo el mundo visible» ${ }^{13}$. Schelling interpreta la materia en términos de potenciales, intensivos o virtuales de un sustrato capaz de engendrar la existencia en la inmanencia de su seno, tan oscuro e impenetrable como la negatividad sustancial, y a la vez tan activo y autocreador como el sujeto idealista. Su energía inagotable genera y regenera el dinamismo continuo de lo real, en un juego de fuerzas siempre abierto que "vuelve a parirse una y otra vez» ${ }^{14}$. En una palabra, Schelling reemplaza el acto puro trascendente — del dualismo sustancialista- por la potencia inmanente, la luminosidad espiritual por la oscuridad material, el Grund de la causa incausada por un abisal Ungrund autocreador. Con esto, el romanticismo schellingiano se convierte en el precursor del nuevo materialismo.

En el Sistema del idealismo trascendental, Schelling analiza el concepto de materia en referencia al yo trascendental, del cual se diferencia en tanto que objeto absoluto o actualidad real respecto de la acción subjetiva ideal. La materia se presenta allí como categoría nuclear de una física especulativa que la deduce a partir del concepto de fuerza o potencia, y la define en los términos sintéticos de dos fuerzas activas opuestas: una fuerza expansiva infinita y una fuerza negativa, inhibitoria y limitante. La materia media y supera ambas acciones opuestas en la unidad de «una tercera fuerza que sea sintética de ambas y corresponda a la actividad sintética del Yo en la intuición» ${ }^{15}$. Esta unidad sintética de fuerzas que

8 Schelling, F. W. J., Sistema del idealismo trascendental, trad. Jacinto Rivera de Rosales y Virginia López Domínguez, Anthropos, Madrid 2005, p. 255.

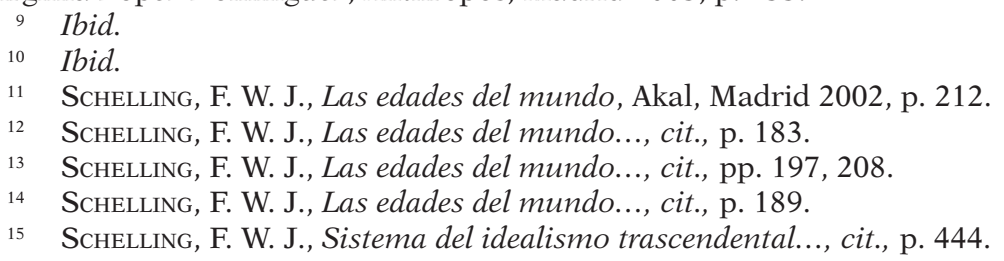


es la materia, se manifiesta físicamente en la gravedad entendida como tendencia universal a lo indiferenciado, que organiza y unifica el mundo natural ${ }^{16}$. De la gravedad derivan los que Schelling describe como los tres grandes dinamismos de la materia, a saber: el magnetismo, la electricidad y el quimismo, resumidos a su vez en el galvanismo. Estos tres grandes procesos materiales tienen su correspondiente subjetivo-trascendental en los actos intelectivos del yo, su correlación se reduce en última instancia a la diferencia del absoluto consigo mismo.

En resumidas cuentas, el romanticismo refleja la restitución de la materia al dinamismo absoluto, el retorno de su consistencia autoactiva, sistematizada por Schelling en la categoría de potencia como negatividad medial. A tales presupuestos especulativos se ceñirá el nuevo materialismo, dialectizado por una materia «nomaterial», que resulta siempre «más-que»o «menos-que» sí misma, pero nunca simple e inmediatamente tal.

\section{LA MATERIA CONCIPIENTE DEL CONCEPTUM HEGELIANO}

En el contexto del idealismo romántico, Hegel merece una consideración especial tanto por el rigor sistemático de su obra, como por su repercusión histórica y su novedoso renacimiento a la luz del giro especulativo contemporáneo. Su pensamiento germina en el suelo del romanticismo y lleva su impronta. Beiser la resume en las siguientes ideas: «el idealismo absoluto de Hegel, su concepción orgánica de la naturaleza, su crítica al liberalismo, sus ideales comunitarios, su spinozismo vitalizado, su concepto de dialéctica, su intento de sintetizar comunitarismo y liberalismo: todas estas ideas suelen ser vistas como propiamente hegelianas, pero son parte de una herencia romántica común ${ }^{17}$. En la cuestión que nos concierne, podríamos decir que Hegel hereda y prolonga el proyecto romántico de concebir la unidad de la vida - la sustancia spinoziana vitalizada por Herderen y por la diferencia del sujeto fichteano en su reflexión autocreadora.

El vitalismo romántico resulta particularmente visible en el Hegel juvenil, donde la categoría de vida lidera la dialéctica de la unión y la no unión, de la oposición y la relación ${ }^{18}$. Lo que para el primer Hegel significa dinamismo medial de la vida, el Hegel maduro lo interpretará en los términos del concepto, ese «simple pulso vital» ${ }^{19}$ de todas las cosas que constituye la acción de la identidad y la no identidad, la diferencia y negación respecto de sí mismo, o bien, la «actividad que se media consigo misma» ${ }^{20}$. A imagen y semejanza de la vida, el concepto abre todas las venas de lo real para verse

16 Cf. Schelling, F. W. J., Escritos sobre filosofía de la naturaleza, trad. Arturo Leyte, Alianza, Buenos Aires 1996; también Grant, Iain Hamilton, Philosophies of Nature after Schelling, Continuum, New York 2006.

17 Beiser, Frederick C., Hegel, Routledge, New York \& London 2005, p. 35.

18 Cf. Hegel, Georg. W. F., Fragmento de sistema de 1800, en Escritos de juventud, trad. José M. Ripalda, FCE., México 1978, p. 401.

19 Hegel, G. W. F., Ciencia de la Lógica, trad. A. y R. Mondolfo, Solar - Hachette, Buenos Aires 1968, p. 36.

20 Hegel, G. W. F., Enciclopedia de las ciencias filosóficas en compendio: para uso de sus clases, trad. R. Valls Plana, Alianza, Madrid 2005, § 338. 
brotar por ellas y su inmanencia garantiza la organicidad del todo, en el que cada miembro disuelve su independencia abstracta por la mediación concreta de la totalidad.

En consonancia con Schelling, la materia hegeliana es comprendida en el seno del dinamismo absoluto y de aquí su consistencia especulativa en tanto que diferencia primera, inmediata y exterior, que es pura negatividad conceptual. Como esa noche indiscernible en la que el absoluto se recoge a sí mismo, así la materia lo contiene todo en su indiferencia conceptiva. Ya la Filosofía real de Jena describía la materia absoluta o éter como el ser simple, puro concepto en sí o pura negatividad que lo concibe todo y es por eso «simplemente la materia grávida, la cual, como movimiento absoluto, es en sí la fermentación ${ }^{21}$. La idea de la materia como intimidad reflexiva del concepto, pura y simple negatividad conceptual, continuará en la Lógica de la esencia ${ }^{22}$ en los términos de esa simple identidad indiferenciada de la reflexión que funda la ex-sistencia.

La consistencia negativa de la materia — que el análisis de la Lógica conserva en su elemento puramente ideal- despliega en la Filosofía de la naturaleza todo su contenido concreto, porque allí la materia asume la exterioridad espacio-temporal del concepto. La filosofía de la naturaleza hegeliana descubre la materia en su transición de lo ideal a lo real como negatividad medial efectiva, realizada en una existencia concreta que es siempre para Hegel el aquí y ahora del concepto. En líneas generales, de la filosofía de la naturaleza se desprenden algunos rasgos centrales de la materia que podríamos resumir en los siguientes: exterioridad, fuerza/ movimiento y mediación. A tales determinaciones nos referiremos brevemente.

La exterioridad de la materia constituye la ex-posición del concepto en y por la unidad sintética espacio-temporal que la determina y tensiona internamente ${ }^{23}$. La materia es lo exterior tanto con respecto a la intimidad ideal que ella realiza en las dimensiones del espacio y el tiempo, como con respecto a su propia identidad material, pospuesta por la diferencia temporal y desgarrada en partes extra partes que se oponen entre sí: elementos extra elementos, multiplicidad, diversidad y gradación de entes extra entes. En ambos respectos, podría decirse que la materia es en sí misma exterioridad, ponerse afuera de la idea en el elemento espaciotemporal que multiplica las diferencias, o bien, poner su propio centro fuera de sí $^{24}$. En la tensión entre su ser-afuera diseminado y su centro de unidad interior, la materia define la segunda de sus determinaciones, a saber: su fuerza semoviente.

Si el ser exterior constituye la identidad inmediata de la materia, la fuerza determina su reflexión interior ${ }^{25}$ : esa íntima tensión que la niega y expulsa afuera, y que Hegel denomina — haciéndose eco de la física newtoniana- «gravedad». En tanto que categoría de la reflexión, la fuerza es referencia negativa a sí, autorrepulsión e impulso hacia lo otro. La Fenomenología ${ }^{26}$ describe la fuerza como determinante

${ }_{21}$ Hegel, G. W. F., Filosofía real, trad. José María Ripalda, FCE, México 2006, p. 5.

22 Hegel, G. W. F., Ciencia de la Lógica..., cit., pp. 397-400, 432-438; Enciclopedia..., cit., $\S 125-130$.

23 Hegel, G. W. F., Enciclopedia..., cit., § 261.

24 Hegel, G. W. F., Enciclopedia..., cit., § 389, 262.

25 Hegel, G. W. F., Enciclopedia..., cit., § 261-262; también Filosofía real..., cit., p. 34.

26 Hegel, G. W. F., Fenomenología del espíritu, trad. W. Roces, México, Fondo de Cultura Económica, México 1966, pp. 82-88. 
material correlativo al entendimiento finito y constituido por el desdoblamiento de dos movimientos contrarios: expansión-exteriorización y contracción-retorno sobre sí. A fuerza de este doble movimiento, la materia construye su identidad reflexiva, o bien, su ser-sujeto de la propia negación en el continuo desgarramiento del aquí y ahora, a través del cual vuelve sobre sí. Este carácter subjetivo del ser material, el Hegel de Jena lo describía en los términos de un «movimiento inmediato, de vuelta en sí, libre, autónomo, que es simple consistencia igual a sí misma ${ }^{27}$. La materia es así, no mera sustancia, sino sujeto reflexivo.

El auto-movimiento material es el sujeto mismo en ese desdoblamiento de extraversión y retorno que Hegel llama en sentido estricto «mediación» y que convierte a la materia en una realidad medial. Que la materia sea mediación significa que ella es en sí su propia negatividad, en y por la cual deviene forma, espíritu, cultura. Respecto de estas últimas configuraciones, podría decirse lo mismo que Hegel aplica a la forma, a saber, que a través de ella la materia decide su propia determinación inmanente, su ser-dentro-de-sí en el cual subsiste negativamente ${ }^{28}$. Forma, espíritu, cultura emergen de la materia como alteridad determinante de sí misma y lo hacen según el dinamismo ascendente de un proceso continuo, en el cual cada configuración asume, niega y supera la precedente, al modo de un organismo en constante crecimiento.

En cierto sentido, Hegel —al igual que el idealismo romántico representado por él- prolonga aquella vieja tradición de la materia como sustrato negativo, sub-jectum infinito e indeterminado. Sin embargo, en un sentido más profundo, él rompe completamente con ella al sostener la consistencia autoactiva de la negación en y por la inmanencia medial del fundamento. La negatividad material resulta así potencia conceptiva de la realidad, ya natural, ya espiritual, ya conceptum. Esta capacidad concipiente revela la auténtica esencia especulativa de la materia, que no es sino la esencia misma del absoluto romántico-idealista, a saber: la libertad creadora. Porque la materia especulativa no es simplemente sustancia sino medialmente sujeto, su actualidad se reduce en última instancia a la libertad, esa potencia viva y creadora por antonomasia del idealismo, que emerge así en el trasfondo de una materialidad conceptiva.

El dinamismo creador —concipiens/conceptum - de la materia hegeliana responde a su libertad esencial y de aquí las dos grandes consecuencias de la especulación romántico-idealista respecto de la naturaleza. En primer lugar, la superación de la relación causal empírica con su posición tautológica del efecto, por la espontaneidad de un fundamento circular y retroactivo a la creación del efecto. En segundo lugar, la elevación del mundo material desde su pasiva y muda sujeción a la acción humana, hacia la agencia inteligible y autoactiva de la libre concepción significante. Esta doble consecuencia del idealismo en general y del hegelianismo en particular los pone a la vanguardia del giro materialista contemporáneo, con un "potencial ecológico»" medioambiental y post-humanista renovador de la cultura hegemónica.

27 Hegel, G. W. F., Filosofía real..., cit., p. 31.

28 Cf. Hegel, G. W. F., Ciencia de la Lógica..., cit., p. 399; también Enciclopedia..., cit., §§ 271-272.

29 Stone, A., Petrified Intelligence. Nature in Hegel's Philosophy, SUNY, New York 2005, pp. 168-69. 
El primer programa sistemático del idealismo alemán soñaba con una «física en grande ${ }^{30}$, a la altura de esa libertad creadora descubierta por los tiempos modernos y realizada por la nueva materialidad. Hegel — así como antes Schelling — responde a ese proyecto con una física especulativa cuya especificidad reside en la potencia conceptiva de la materia. Materia y libertad constituyen la acción recíproca de una Bildung universal en continuo crecimiento. Tal es la grandeza de esa física, cuya clave especulativa articula dos conceptos centrales de la ontología contemporánea: la negatividad medial de la materia y la espontaneidad libre de su devenir.

La intimidad reflexiva de la naturaleza en la cual se reconoce la subjetividad romántica, el Abgrund infinito de la potencia schellingiana o la negación medial del concepto de Hegel, cualquiera de ellos describe un nuevo sustrato material, ni pasivo ni receptivo, sino autoactivo e inteligible. Sobre esta materialidad se apoya el giro neo-materialista de la especulación contemporánea, por una suerte de retorno autorreflexivo que cierra el círculo del espíritu en la materialidad de su origen.

\section{El Último PRograma materialista del VIEJo ideALismo}

Lo que Adrian Johnston denomina «el último programa sistemático del idealismo alemán ${ }^{31}$ recupera el proyecto de una gran física, reeditada ahora como un nuevo materialismo especulativo, para el cual la materia es mediación no-material o másque-material. El nuevo materialismo dialéctico se entiende a sí mismo como una radicalización del idealismo ${ }^{32}$, que se ha tomado muy en serio la fuerza conceptiva de la materia en tanto que espontaneidad libre del absoluto inmanente.

La densidad especulativa le permite al nuevo materialismo elevarse por sobre los dos opuestos dualistas: el materialismo vulgar, mecanicista o positivista, para el cual la realidad dada es anterior e independiente de la conciencia humana que la objetiva; y el materialismo histórico, para el cual la realidad material es producto de la acción humana en tu tenor económico-social, cultural o lingüístico ${ }^{33}$. En el medio de la oposición, la materia del materialismo dialéctico es tan real e irreductible como la sustancia inmediata, a la vez que tan mediada y construida como la actualidad libre del sujeto; tan una y simple como aquella, a la vez que tan diferencial y negativa como esta. Los neo-materialistas reeditan la superación de la sustancia por el sujeto en los términos de una materia que deviene lo absoluto autoconceptivo, espontáneo e indecidible. Su clave especulativa retoma la novedad del absoluto idealista, incorporado en la inmanencia perfecta de lo material.

Autores tan diversos como John Caputo, Mark Taylor, Gabriel Markus, Slavoj Žižek, Catherine Malabou o Adrian Johnston, para mencionar solo algunos, comparten la herencia negativa de lo absoluto, junto con la fuerza creadora de

30 Hegel, G. W. F., Primer programa de un sistema del idealismo alemán, en Escritos de juventud..., cit., p. 219.

31 Johnston, A., Adventures..., cit., p. 23, 13.

32 Cf. ŽıžEK, S., Absolute Recoil. Towards A New Foundation of Dialectical Materialism, Verso, London \& New York 2014, p. 31.

33 Cf. Timpanaro, S., Sul materialismo, Nistri-Lischi, Pisa 1970, pp. 16 ss. 
la negación. La promoción de lo negativo a primer principio o fundamento del orden ontológico se traduce en una realidad que es siempre y en todo caso "noTodo ${ }^{34}$. Supuesto como origen y fundamento, lo negativo - noche oscura, caos o Ungrund abismal- procede en el sentido de una des-fundamentación de lo real, que el idealismo asume en la circularidad de un principio retroactivo al efecto, y el materialismo recoge como incompletitud, desajuste o ruptura de lo absoluto. Habida cuenta de la negatividad material, entonces —explica Žižek- para el materialismo dialéctico «el no-Todo es materia» ${ }^{35}$, lo cual es otro modo de enunciar la no-materialidad de la materia misma, su diferencia y contradicción, de la cual emerge la multiplicidad del orden real, simultáneamente material e inmaterial. La diferencia material-inmaterial inscrita en el seno de la materia misma reproduce ese desdoblamiento especulativo, que antes les aseguraba a los románticos la unidad vital de la unión y la no-unión.

La negatividad material no es la nada simple e inmediata del puro ser trascendente, sino la negación reflexiva y medial de lo sido inmanente, de donde resulta su consistencia dialéctica y afirmativa. Žižek la llama en este sentido una nada «menos que nada» ${ }^{36}$, es decir, entre el ser puro y la pura nada, en medio de los cuales se ubica lo posible o potencial. Adrian Johnston se refiere a una «negatividad materializada ${ }^{37}$, más que simplemente negativa y menos que totalmente material, cuya acción es multiplicadora de diferencias, contradicciones y devenires espaciotemporales. La negación absoluta de la materia produce las incontables «diferencias mínimas» ${ }^{38}$ del mundo finito y contingente, siempre en tensión, desajuste o paralaje consigo mismo.

Afirmada de manera medial entre el puro ser y la pura nada, la negatividad material cumple la función dialéctica de continuidad y ruptura, identidad y diferencia propia del devenir. Su virtualidad infinita e inagotable garantiza la permanencia sustancial de la realidad, mientras que la espontaneidad autocreadora del sujeto asegura su novedad y contingencia. El nuevo materialismo relee el despliegue conceptual del absoluto como la continua novedad de una potencia infinita irreductible a su acción, espontánea respecto del orden causal finito, y negativa en relación con cualquier positividad fundante. De la negatividad potencial del origen, el materialismo dialéctico deriva la novedad radical de la existencia finita y contingente, reconducible desde el punto de vista especulativo a la libertad de un origen espontáneamente autoactivo. La libertad se dice de la materia en el mismo sentido en que el sujeto se dice de la sustancia, como su negatividad creadora, medialmente idéntica y la misma.

En los párrafos que siguen, quisiéramos mostrar a título de ejemplo el modo en que dos representantes de este nuevo materialismo asumen y tornean el absoluto idealista. Nos referimos en concreto a Slavoj Žižek y Catherine Malabou, para

34 Bryant, L., Srnicer, N. y Harman, G. (eds.), The Speculative Turn: Continental Materialism and Realism, Re.Press, Melbourne 2011, p. 407; ŽıžEK, S., Absolute Recoil..., cit., p. 1.

35 ŽıžEK, S., The Parallax View, MIT Press, Cambridge 2006, p. 168.

36 ŽIžEK, S., Absolute Recoil..., cit., pp. 385, 391.

37 ŽıžEK, S., Crockett, Clayton,,Davis, Creston (eds.), Hegel and the Infinite, Columbia University Press, New York 2011, pp. 159 ss.

38 ŽıžEK, S., The Parallax View..., cit., p. 11. 
quienes el concepto de materia satisface tanto las determinaciones lógicas de la idea, como las determinaciones reales del devenir efectivo, ya natural, ya espiritual. La materia traspone la virtualidad conceptiva del concepto - natura naturans- a la realidad efectiva - natura naturata-, en el amplio sentido de una naturaleza des-naturalizada o espiritual, siempre más y menos que sí misma.

El pensamiento de Žižek profesa el idealismo de un absoluto no-absoluto y, por lo tanto, continuamente quebrado, contradicho, desajustado consigo mismo. En el caso de Schelling, Žižek encumbra Las edades del mundo a "texto fundacional del materialismo dialéctico ${ }^{39}$ por el hecho de haber allí una analítica de la finitud y temporalidad anterior - e incluso superior - a la proclamada por el posthegelianismo. El determinante ontológico que domina el materialismo schellingiano tal como lo interpreta Žižek, reside en la abisal oscuridad del fundamento de la existencia, potencialidad infinita que media entre el no-ser y el ser. Schelling invierte el fundamento luminoso, actual y trascendente de la tradición filosófica en un Ungrund caótico, potencial e inmanente, a través del cual retorna el vacío primordial de una mítica inmemorial. La noche más remota de todos los tiempos, de cuyo seno todo nace y al que todo retorna en su disolución, reaparece en los tiempos modernos bajo la clave especulativa de una potencia infinita, capaz de poder y no-poder. La síntesis idealista de Žižek relee el carácter medial de la potencia schellingiana en los términos hegelianos de una doble negación: «imposibilidad redoblada ${ }^{40}$ de sí mismo y lo otro, resuelta en la virtualidad diferencial infinita de la finitud.

La negatividad medial que define el Ungrund schellingiano, determina también según Žižek el esencial desgarramiento del sujeto hegeliano ${ }^{41}$, sive la materia. Žižek homologa el sujeto de Hegel con el no-todo material, ambos definidos por la negatividad reflexiva, dinámica e inmanente del absoluto idealista, del cual emerge —ex nihilo- la realidad efectiva. En palabras de Žižek, «el Concepto mismo es no-todo» ${ }^{42}$ y de aquí su continua concepción material. En el caso del autodespliegue conceptual que Hegel propone, la interposición de la nada, menosque-nada, como medio del devenir garantiza para Žižek la novedad creadora y la contingencia de lo real, de manera tal que la necesidad inmanente del concepto se identifica con la espontaneidad de su acción libre. La libertad del sujeto hegeliano operaría entonces como des-fundamento de lo real y reaseguro de su contingencia, de donde su sistema no implicaría la reproducción tautológica causa-efecto, sino la espontaneidad creadora «de lo eternamente Nuevo en el proceso del devenir» ${ }^{43}$.

A la posible objeción anti-materialista o espiritualista de un eventual purismo hegeliano, Žižek se adelanta con la paráfrasis de la Fenomenología: «el espíritu es

39 ŽIžEK, S., The Abyss of Freedom. Ages of the World, The University of Michigan Press, 1997, p. 33; cf. también ŽıžEK, S., The Invisible Remainder. On Schelling and Related Matters, Verso, London \& New York 1996, pp. 13 ss.

40 ŽıžEK, S., Absolute Recoil..., cit., p. 344.

41 Cf. ŽıžEK, S., The Abyss of Freedom..., cit., p. 7.

42 ŽıžEK, S., The Sublime Object of Ideology, Verso, London \& New York 1989, p. 6.

43 ŽıžEK, S., Órganos sin cuerpo. Sobre Deleuze y consecuencias, Pre-textos, Valencia 2004, pp. 30-31. 
un hueso ${ }^{44}$, y lo «es» al modo de una identidad ni simple ni inmediata, sino refleja, medial y conceptiva. La cópula de un juicio infinito —el espíritu «es» un huesosupera la identidad abstracta y representativa de los términos para elevarse al dinamismo de la especulación, donde la identidad resulta el tercer término de la mediación, cuya negatividad se dice ya materia, ya sujeto, ya absoluto.

En esta misma línea argumental, se comprende la afirmación según la cual «solo el materialismo puede explicar exactamente los fenómenos del espíritu, de la conciencia, etc. ${ }^{45}$, en tanto y en cuanto se trata aquí de una materia conceptivamente no-material, es decir, concipiente de lo otro. Las partes extra partes en las cuales la materia se diferencia y afirma, que en sentido estricto se aplica a la exterioridad cuantitativa de la masa, en sentido amplio podría aplicarse a la emergencia cualitativa de fenómenos anímicos y espirituales, por los cuales la exterioridad material se diferencia a fin de volver sobre sí misma en su propio reconocimiento. El desdoblamiento no-material, más-que- o trans-material coincide con el despliegue progresivo del espíritu por una suerte de trascendencia inmanente a la materia misma. Por eso, los fenómenos anímicos y espirituales no resultan externos sino interiores a la identidad material.

También Catherine Malabou propone su materialismo dialéctico en continuidad con el idealismo, en especial hegeliano, y articulado en torno al concepto de "plasticidad», el otro nombre de la negación medial. La categoría de plasticidad es definida por su autora como «la unidad originaria de acción y ser actuado, de espontaneidad y receptividad. Medio en la diferenciación de los opuestos, la plasticidad mantiene los extremos juntos en su acción recíproca, habilita la función de una estructura de anticipación donde los tres términos del proceso temporal son articulados: la síntesis originaria, la hipotiposis o corporeización de lo espiritual, la relación de los momentos del tiempo ${ }^{46}$. La plasticidad malabouniana reitera los rasgos centrales de la materia, a saber: originalidad indeterminada, potencialidad medial, actualización recíproca de la diferencia y circularidad retroactiva del fundamento. Ella subsiste en su continua transformación espacio-temporal, exterior a sí misma e inmanente a todas las cosas, incluso al devenir temporal de los fenómenos conscientes. A imagen y semejanza de la elasticidad hegeliana ${ }^{47}$, lo plástico mantiene la continuidad orgánica en la diferenciación procesual. En el mismo sentido, la plasticidad satisface las determinaciones distintivas del absoluto idealista: inmanencia y diferencia radical. Ahora bien, cuando esta diferencia debe ser concebida en aquella inmanencia, entonces el único otro posible es el otro de sí mismo, con la doble valencia conceptiva y contingente de la alteridad.

La plasticidad subyacente a lo real justifica su contingencia y accidentalidad, porque responde a la espontaneidad autocreadora del origen que ninguna causalidad de orden finito agota. En la raíz de toda causa empírica reside la espontaneidad de un origen libremente auto-actuante, del cual deriva la contingencia del

${ }^{44}$ Žıžex, S., The Parallax View..., cit., p. 5; Absolute Recoil..., cit., p. 332; Hegel, Georg. W. F., Fenomenología..., cit., pp. 205-06.

45 Žı̆̌EK, S., The Parallax View..., cit., p. 167.

46 Malabou, C., The Future of Hegel. Plasticity, Temporality and Dialectic, trad. Lisabeth During, Routledge, New York 2005, p. 186.

47 Cf. Hegel, G. W. F., Enciclopedia..., cit., § 298. 
devenir. Esta última no se mide por el azar o la necesidad causal empírica, sino por la sobredeterminación libre del origen meta-físico. Malabou se refiere en este sentido a la producción de un «evento material» ${ }^{48}$ por el cual adviene la materia en su diferencia no-material, que resulta forma y vida, espíritu y cultura. El evento material conserva tanto la disrupción de lo nuevo como la continuidad medial de lo que la autora denomina "plasticidad intermedia» $\mathrm{O}$ "plasticidad-enlace» ${ }^{49}$, capaz de mediar la transición de lo mismo a lo otro, de la identidad a la diferencia. En el caso concreto del advenimiento material de la vida al cuerpo, o de la autoconciencia espiritual a lo anímico, Malabou interpreta el sustrato físico como una suerte de "proto-sí mismo» ${ }^{50}$ del cual emerge la alteridad sensitiva y espiritual por la reflexión negativa de la materia misma. El corolario final de esta plasticidad impredeciblemente transformadora es extender la espontaneidad libre y la temporalidad histórica a todo el orden natural. También la naturaleza tiene para Malabou una historia y un acontecer creador, independiente de la acción humana y correspondiente con su origen material.

Este tipo de materialismo plástico, dialéctico y medial es el que Malabou le atribuye al idealismo hegeliano y a su respecto comenta: «Hegel es el primer filósofo que ha convertido la palabra plasticidad en un concepto y que desarrolló una teoría de las relaciones entre naturaleza y mente conflictiva y contradictoria en esencia. Releer su filosofía de la naturaleza puede enseñarnos mucho sobre la transición de lo biológico a lo espiritual, sobre el modo en que la mente es ya sí-mismo (Selbst), espíritu-naturaleza, en cuyo corazón las diferencias son todas a la vez físicas y psíquicas ${ }^{51}$. A la sazón, la autora recuerda ese pasaje de la Enciclopedia ${ }^{52}$ donde Hegel afirma la identidad esencial —es decir, conceptual—de lo físico y lo espiritual, cuya mediación resuelve una de las preocupaciones centrales de su pensamiento, a saber, la transformación de la existencia natural en ser histórico y especulativo.

Sin embargo, si hay algún punto en el que este nuevo materialismo parecería rectificar la clásica lectura idealista, ese punto consiste en la posición privilegiada del espíritu humano por encima del orden natural, jerárquicamente subordinado a la acción humana autoconsciente. El privilegio de la filosofía del espíritu por sobre la filosofía de la naturaleza, entendidas ambas en términos opuestos y excluyentes, resultaría el talón de Aquiles de un idealismo piramidalmente edificado. Ante tales privilegios espirituales, el giro materialista insiste en un espíritu no-espiritual, menos-que la totalidad, reduplicado en redes horizontales, omnicentrado y siempre aferrado a su origen material. La propuesta de Malabou de una «filosofía del espíritu naturalista ${ }^{53}$ podría interpretarse como una radicalización del idealismo, que intenta cerrar el círculo especulativo en el principio material del espíritu mismo. Y esto vale no solo en el sentido lógico de la inmanencia más cabal ni en

48 Malabou, C., The New Wounded. From Neurosis to Brain Damage, trad. Steven Miller, Fordham University Press, New York 2012, p. 212.

49 Malabou, C., What Should We Do with Our Brain?, trad. Sebastian Rand, Fordham University Press, New York 2008, p. 69.

50 Malabou, C., What Should We Do..., cit., p. 59.

51 Malabou, C., What Should We Do..., cit., p. 80.

52 Cf. Hegel, G. W. F., Enciclopedia..., cit., § 396.

53 Malabou, C., The New Wounded..., cit., p. XII. 
el sentido naturalista de un panteísmo cósmico, sino particularmente en el sentido antropológico, psicológico, ético, político, estético y religioso de un espíritu que se reconoce en su materialidad. Que la materia, el cuerpo, la vida sean la última palabra ética, política y religiosa, determina el contragiro ontológico del nuevo idealismo en su retorno sobre sí.

\section{A MODO DE CONCLUSIÓN}

Una vez que el idealismo absoluto dejó atrás la trascendencia perfecta e inmutable del acto puro, respecto del cual la materia funcionaba como sustrato pasivo, receptivo y degenerante, entonces la inmanencia del origen no pudo ser menos-que material y la materia, menos-que la potencia vital conceptiva del todo. Lo novedoso de este absoluto - que lo distingue de la clásica potencia pura, incapaz de no poder- es el hecho de poder no-poder y de hacerlo por su propia negatividad reflexiva. Este absoluto partido, desdoblado y conceptivo es la materia.

Más viejo o más nuevo, la verdad del materialismo idealista es que no hay nada, ni el cielo ni en la tierra, que no sea a la vez material y no-material, unidad de lo mismo y lo otro, traspasar de la simple inmediatez a la negación del devenir. La no-materialidad de la materia misma puede tener muchos nombres: logos, libertad, conciencia, espíritu, cultura, historia, etc., todos ellos concebidos en la inmanencia del sustrato que es además sujeto. La elaboración de la sustancia en términos de sujeto, o de la materia en términos de actualidad libre, se traduce en un universo no todo-natural y no todo-espiritual, que ha perdido el sentido jerárquico de la diferencia para apoyarse en la pura reciprocidad de su incompletitud.

La resignificación neo-materialista del universo, en sintonía con las nuevas corrientes de pensamiento ecológico, medioambiental, post-humanista y biocivilizatorio, retoma el viejo tópico de la tradición filosófica que recomienda el actuar humano secundum naturam, con la salvedad de que se trata ahora de una naturaleza no-material o más-que-natural. La acción humana secundum naturam se inscribe en la inmanencia de un poder espontáneamente creador de nuevas configuraciones vitales, multiplicadas en redes, puentes y circuitos de mediación. Una cultura espiritual a la altura de esta restitución material expresa el entusiasmo y la veneración de un mundo lleno de dioses, saturado de vida sobrenatural.

El desiderátum idealista de construir el reino de Dios en la Tierra atraviesa hoy la radicalización especulativa de construir el reino del espíritu en la materia, de concebir el logos de los cuerpos, humanos y no humanos, y sostenerlos mutuamente en acciones reciprocas. El último programa sistemático propone hoy la liberación de lo divino al dinamismo superior de la vida material.

\section{BiBLIOGRAFÍA}

Béguin, A. (1978). El alma romántica y el sueño, trad. Mario Monteforte Tolero. Madrid: FCE. Beiser, F. C. (2005). Hegel. New York \& London: Routledge.

- (2003). The Romantic Imperative. The Concept of Early Romanticism. Cambridge \& London: Harvard University Press. 
Bennet, J. (2010). Vibrant Matter. A Political Ecology of Things. Durham \& London: Duke University Press.

Bryant, L., Srnicek, N. y Harman, G. (eds.) (2011). The Speculative Turn: Continental Materialism and Realism. Melbourne: Re.Press.

Dunham, J., Grant, I. H. y Watson, S. (2011). Idealism. The History of a Philosophy. Montreal \& Kingston: McGill-Queen's University Press.

Gabriel, M. (2015). Fields of Sense: A New Realist Ontology, Edinburgh University Press.

Gabriel, M. y Rasmussen, A. M. (2017). German Idealism Today. Berlin \& Boston: De Gruyter.

Grant, I. H. (2006). Philosophies of Nature after Schelling. New York: Continuum.

Gratton, Peter (2014). Speculative Realism. Problems and Prospects. London \& New York: Bloomsbury.

Hegel, G. W. F. (2006). Filosofía real, trad. José María Ripalda. México: FCE.

- (2005). Enciclopedia de las ciencias filosóficas en compendio: para uso de sus clases, trad. R. Valls Plana. Madrid: Alianza.

- (1978). Escritos de juventud, trad. José M. Ripalda. México: FCE.

- (1968). Ciencia de la Lógica, trad. A. y R. Mondolfo, Solar. Buenos Aires: Hachette.

- (1966). Fenomenología del espíritu, trad. W. Roces, México: Fondo de Cultura Económica

Houlgate, S. (ed.) (2011). A Companion to Hegel. Oxford: Blackwell Publishing.

- (1998). Hegel and the Philosophy of Nature. Albany: SUNY.

Johnston, A. (2014). Adventures in Transcendental Materialism. Dialogues with Contemporary Thinkers. Edinburgh University Press.

- (2008). Zizek's Ontology. A Trascendental Materialist Theory of Subjectivity. Northwestern University Press.

Kirby, V. (ed.) (2017). What if Culture was Nature all Along?. Edinburgh: University Press, Edinburgh.

Malabou, C. (2012). Ontology of the Accident. An Essay on Destructive Plasticity, trad. Carolyn Shread. Cambridge-Malden: Polity.

- (2012). The New Wounded. From Neurosis to Brain Damage, trad. Steven Miller. New York: Fordham University Press.

- (2008). What Should We Do with Our Brain?, trad. Sebastian Rand. New York: Fordham University Press.

- (2005). The Future of Hegel. Plasticity, Temporality and Dialectic, trad. Lisabeth During. New York: Routledge.

Novalis (1948). Los fragmentos. Los discípulos en Sais, introd. y trad. Mauricio Maeterlinck. Buenos Aires: El Ateneo.

O’ Neill Burns, M. (2016). «Vitality or Weakness», en Angelaki. Journal of Theoretical Humanities, 21/4, pp. 11-22.

Schelling, F. W. F. (2005). Sistema del idealismo trascendental, trad. Jacinto Rivera de Rosales y Virginia López Domínguez. Madrid: Anthropos.

- (2002). Las edades del mundo. Madrid: Akal.

- (1996). Escritos sobre filosofía de la naturaleza, trad. Arturo Leyte. Buenos Aires: Alianza.

- (1989). Investigaciones filosóficas sobre la esencia de la libertad humana y los objetos con ella relacionados, trad. $\mathrm{H}$. Cortés - A. Leyte. Barcelona: Anthropos.

Schlegel, F. (1968). Dialogue on Poetry and Literary Aphorism, trad. e intr. Ernst Behler - Roman Struc. Pennsylvania: The Pennsylvania State University Press

Stone, A. (2005). Petrified Intelligence. Nature in Hegel's Philosophy. New York: SUNY.

Timpanaro, S. (1970). Sul materialismo. Pisa: Nistri-Lischi.

Trías, E. (2006). La edad del espíritu. Barcelona: DeBolsillo.

Žižek, S. (2014). Absolute Recoil. Towards A New Foundation of Dialectical Materialism. London - New York: Verso.

- (2012). Less than Nothing. Hegel and the Shadow of Dialectical Materialism. London - New York: Verso. 
- (2006). The Parallax View. Cambridge: MIT Press.

- (2004). Órganos sin cuerpo. Sobre Deleuze y consecuencias. Valencia: Pre-textos.

- (1997). The Abyss of Freedom. Ages of the World, The University of Michigan Press.

- (1996). The Invisible Remainder. On Schelling and Related Matters. London - New York: Verso.

- (1989). The Sublime Object of Ideology. London - New York: Verso.

Žižek, Slavoj, Crockett, Clayton, Davis y Creston (eds.) (2011). Hegel and the Infinite. New York: Columbia University Press

Žižek, Slavoj, y Gabriel, Markus (2009). Mythology, Madness and Laughter. Subjectivity in German Idealism. New York: Continuum.

Investigadora Adjunta del CONICET - Argentina

María J. BinetTi

mjbinetti@gmail.com

[Artículo aprobado para publicación en diciembre de 2016] 